\title{
Review Article \\ Effects of HBV Genetic Variability on RNAi Strategies
}

\author{
Nattanan Panjaworayan ${ }^{1}$ and Chris M. Brown ${ }^{2}$ \\ ${ }^{1}$ Department of Biochemistry, Faculty of Science, Kasetsart University, Bangkok 10900, Thailand \\ ${ }^{2}$ Department of Biochemistry, University of Otago, Dunedin 9016, New Zealand
}

Correspondence should be addressed to Chris M. Brown, chris.brown@otago.ac.nz

Received 31 January 2011; Accepted 15 April 2011

Academic Editor: Patrick Soussan

Copyright ( $) 2011$ N. Panjaworayan and C. M. Brown. This is an open access article distributed under the Creative Commons Attribution License, which permits unrestricted use, distribution, and reproduction in any medium, provided the original work is properly cited.

RNAi strategies present promising antiviral strategies against HBV. RNAi strategies require base pairing between short RNAi effectors and targets in the HBV pregenome or other RNAs. Natural variation in HBV genotypes, quasispecies variation, or mutations selected by the RNAi strategy could potentially make these strategies less effective. However, current and proposed antiviral strategies against HBV are being, or could be, designed to avoid this. This would involve simultaneous targeting of multiple regions of the genome, or regions in which variation or mutation is not tolerated. RNAi strategies against single genotypes or against variable regions of the genome would need to have significant other advantages to be part of robust therapies.

\section{RNA Interference as an Antiviral Strategy}

RNA interference (RNAi) is a sequence-specific mechanism to downregulate gene expression. Several pioneering studies have demonstrated the effectiveness of using siRNAs for treating viral diseases caused by HIV, hepatitis $\mathrm{C}$ virus (HCV), and HBV [1-5].

Clinical trials with RNAi have now begun for several disorders, but challenges such as off-target effects, toxicity, and safe and efficient delivery methods have to be overcome before the widespread use of RNAi as a gene-based therapy $[6,7]$. For hepatitis B virus (HBV) several approaches have been taken using various design and delivery strategies with good initial success (reviewed in $[4,5,8,9]$ ) and some limitations [10-12].

Several studies have tested the effect of variability in HBV viral genomes on effectiveness of this antiviral strategy; see $[7,13,14]$ and references therein. This paper will outline the RNAi pathway, current delivery methods, current RNAi design strategies, and the effects of variation on these strategies.

\section{The Mechanism of RNAi}

RNAi is initiated by short double-stranded RNAs (dsRNAs) that lead to the sequence-specific inhibition of their homol- ogous RNAs [15-17]. In the case of HBV, this includes the $3.6 \mathrm{~kb}$ pregenomic RNA (pgRNA), although some targets are within multiple overlapping viral RNAs.

Two major types of RNA have been channeled into the RNAi pathway small interfering RNAs (siRNAs) and microRNAs (miRNAs) by using synthetic dsRNAs or DNA vectors (Figure 1). The siRNAs have a characteristic twonucleotide $3^{\prime}$ overhang, which are processed from larger dsRNAs by Dicer. They are incorporated into RISC, and the sense strand of the siRNA is removed [18-20]. Some studies using HBV have designed siRNAs (and miRNAs) to promote this asymmetric loading of the RISC complex. The antisense strand of the siRNA base pairs with its target RNA, with exact complementarity, and then RISC mediates cleavage and subsequent degradation of the target RNA [21-23] (Figure 1). Perfect base pairing between the siRNA and HBV RNA is a hallmark of siRNA effects, and single base substitutions in the target, due to genome variability, would disrupt this mode of action $[4,8,17,24]$.

Strategies based on miRNAs require engineering genes encoding longer primary transcripts (pri-miRNA based on miRNA genes) that are then processed into 60-70 base paired precursor miRNAs (pre-miRNAs) by the microprocessor complex $[25,26]$. Following processing, the pre-miRNA is exported to the cytoplasm by the Ran-GTP-dependent cargo 


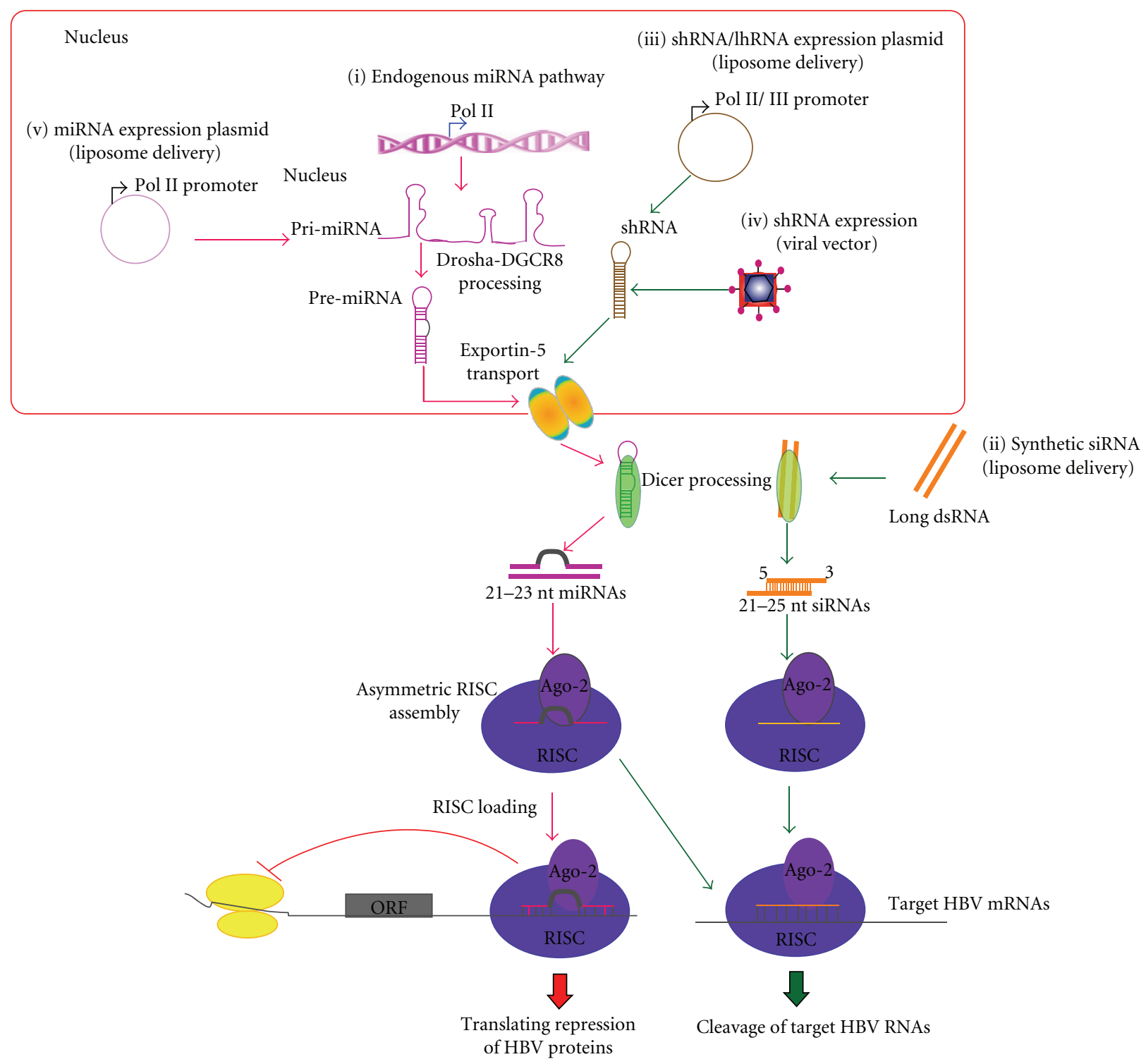

FIGURE 1: RNAi pathways in HBV research. Flow diagram of the miRNA pathway (i) is shown using red arrows, whereas the siRNA pathway is indicated using green arrows. Current RNAi strategies including delivery approaches (ii)-(v) are demonstrated.

transporter Exportin-5 [27]. In the cytoplasm pre-miRNA is processed by Dicer into the mature miRNA, which is incorporated into RISC $[4,8,17,24]$ which targets the viral RNA [28]. Typical cellular miRNAs are not perfectly matched to their mRNA targets, and studies have indicated that they mainly exert silencing through translational repression, rather than degradation [29, 30] (Figure 1). However, later studies indicate that mismatched miRNA-mRNA duplexes can also trigger degradation [31,32]. This may indicate that miRNAs targeted against the HBV pgRNA could also reduce levels of that RNA, rather than just its translation.

\section{RNAi Delivery Mechanisms}

In order to use RNAi-based systems to target viral mRNAs, several delivery strategies have been developed. The two main current strategies are chemically synthesized siRNA duplexes and DNA-based expression cassettes that subsequently generate functional siRNAs in cells. These RNAs are usually short hairpin RNAs (shRNAs) or primary miRNAs (primiRNAs).

Synthetic siRNA duplexes are usually delivered into cells via the endosomal pathway by cationic liposomes, whereas DNA-based expression cassettes require facilitating carriers such as liposomes or viral vectors (Figure 1). Synthetic siRNA duplexes have some limitations in vivo-rapid liver clearance, lack of target specificity, and expense [33-35]. To improve in vivo stability of siRNA duplexes, the backbone of siRNA may be chemically modified and linked to molecules such as $2^{\prime} \mathrm{F}, 2^{\prime} \mathrm{O}-\mathrm{Me}$, and $2 \mathrm{H}[36,37]$.

DNA-based viral expression cassettes may provide costeffective approaches for HBV treatment. Presently, there are 


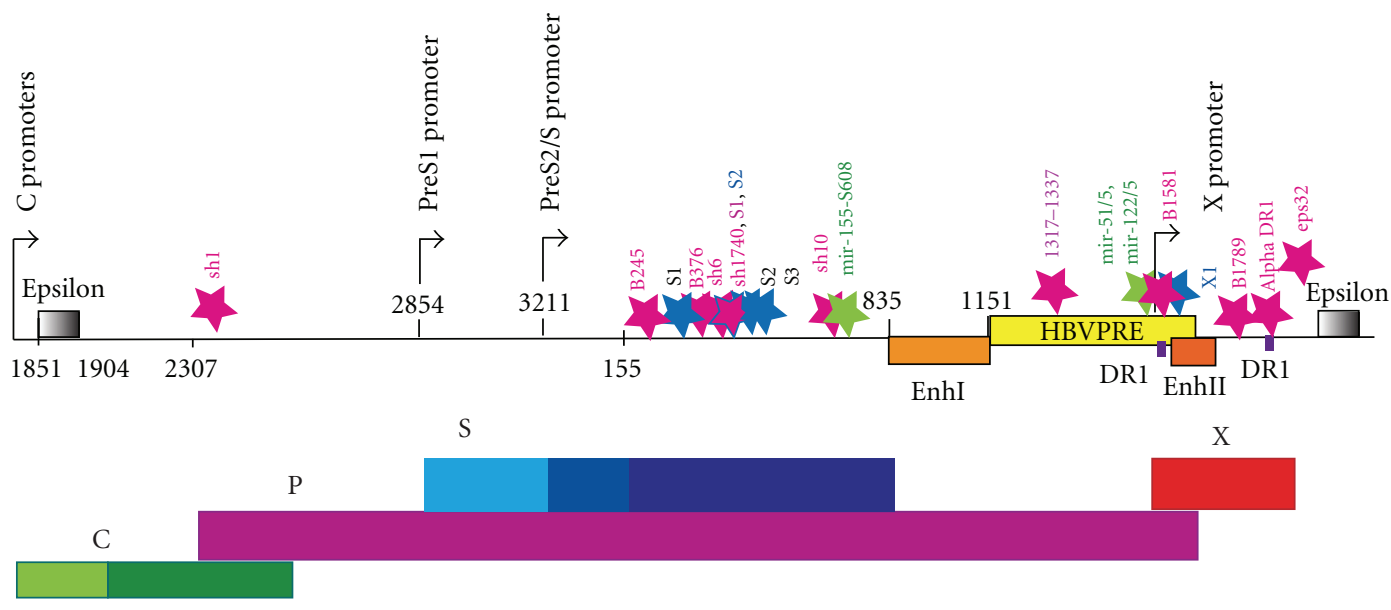

AAA pcRNA/pgRNA

AAA preS1 RNA

AAA preS2/S RNA

AAA X RNA

FIGURE 2: A linear depiction of the HBV genome indicating successful RNAi target sites. Four main promoters (C, S1, S, and X promoters) and regulatory elements such as epsilon, EnhI, EnhII, PRE, and DR1 are indicated on the HBV genome. The four overlapping HBV ORFs are indicated as coloured solid boxes. Thin line represents HBV transcripts. The common poly(A) site is represented as "AAA." Pink stars indicate Group I successful RNAi target sites, while green and blue stars represent Group II and Group III, respectively. The authors designated names of each of the RNA effectors indicated. Numbering indicates nucleotide position, using EcoRI-based numbering system from HBV Genotype A (AM282986, [6]).

a number of viral vectors under development. Each type of viral vector has specific characteristics that need to be determined for the specific target. The adenovirus- and adeno-associated virus- (AAV-) derived vectors provide an efficient delivery vehicle for transient shRNA expression [8]. Particularly, the Ad-gutless vector is used for liver-directed systemic delivery with prolonged silencing effects [38] while a conditionally replicating adenovirus (CRAd) is designed to replicate and kill tumour cells specifically [8]. Retroviruses on the other hand provide major advantage of incorporating the transgenic siRNA genes into the host cell genome for longer-term therapy [39]; other viral vectors have been used $[40,41]$.

\section{Design of RNAi against $\mathrm{HBV}$}

To improve the efficiency of RNAi strategies and limit offtarget effects, several research groups have improved the design of RNAi target sites. Certain characteristics of RNAi target sites contribute to siRNA efficiency; these have been utilised in some rational design approaches, whereas other studies have focused more on conservation of sites in HBV genomes. Specific features that should improve the efficiency of target sites include a UU overhang at the $3^{\prime}$-end $[42,43]$, a $30-50 \%$ GC content, which is effective for the unwinding of the duplex but sufficient for stabilizing interactions between siRNAs and their targets, and the nucleotide at the position 19 should preferentially be an adenine (A) base, as it is naturally found in miRNAs $[8,19,20,44]$. Sun et al. report that there are about 170 sites in the HBV genome that meet simpler minimal criteria for RNAi design-target length 19, GC 35-60 and lack of homopolymer runs [7].

Other considerations relating specifically to RNA polymerase III (Pol III) transcription are that there should be no 4-6 base $T$ tracts within the DNA sequence, because this could act as a termination signal [19]. Importantly, siRNAs must be specific to their target HBV mRNAs and have minimal similarity to cellular mRNA sequences, at least for RNAs expressed in the targeted cells (hepatocytes) to avoid off-target effects.

Results from McCaffrey and Ely et al. indicate that miRNA-based RNAi effectors against HBV pregenomic RNA were more effective than shRNA-based RNAi effectors for the same target sites $[8,24]$. Grimm et al. [11] found that the RNAi toxicity may be caused by competition between the exogenous expressed shRNA and endogenous miRNA for the RNAi machinery (Figure 1). Therefore, features of RNAi effectors are proposed to be similar to cellular miRNAs but not compete detrimentally with it [12]. This might be avoided by strategies using tissue-specific RNA Pol II [45] or weaker Pol III promoters [12].

A complementary rational design is currently proposed to target conserved regions of the HBV genome. This should minimize viral escape that may occur due to selection pressure of RNAi on the target site to mutate $[7,10,13$, 14]. Surprisingly, these include several highly conserved HBV genomic regions that have been demonstrated to be effective target sites for shRNAs despite the presence of known secondary structures (Epsilon, PRE, Figure 2). These 
structures were predicted to reduce the effectiveness of RNAi $[7,14]$.

\section{HBV Genomes to Be Targeted}

The HBV genome contains multiple overlapping DNA, RNA, and protein coding features, meaning that any particular RNAi target sequence is likely to be in more than one transcript. The genome is a partially double-stranded circular DNA of $3.2 \mathrm{~kb}$ that contains four primary open reading frames (ORFs): the core $(\mathrm{C})$, polymerase $(\mathrm{P})$, surface $(\mathrm{S})$, and $\mathrm{X}$, although there may be other protein products $[41,46]$. These ORFs partially overlap each other and are all encoded on the positive strand [47]. Transcription of HBV RNA is initiated by four major promoters- the basal core promoter (BCP), pre S1, preS2/S, and X (Figure 2). These promoters give rise to transcripts that are synthesised in the same direction by host RNA polymerase II. Five major HBV transcripts are known, all are translated. Two sets of $C$ transcripts are initiated at different sites of the $\mathrm{BCP}$ promoter. The longest transcript is the $3.6 \mathrm{~kb}$ precore mRNA (pcRNA). The shorter $\mathrm{C}$ transcript is a pregenomic mRNA (pgRNA) which encodes the $\mathrm{C}$ protein (nucleocapsid protein) and the $\mathrm{P}$ protein. The other three transcripts are preS1, preS2/S, and X, encoding for $\mathrm{S}$ proteins (large surface proteins or preS1), and shorter $\mathrm{S}$ proteins (middle and small $S$ proteins or preS2 and $S$ ) and the $\mathrm{X}$ protein (a transcriptional transactivator), respectively (Figure 2). Therefore, the HBV genome is highly compact and HBV genes are arranged in such a way that many sequences have multiple roles.

Although this compact arrangement restricts plasticity and limits the ability of the virus to mutate, HBV has significant diversity among HBV genotypes [48, 49] and HBV genomes exist as quasispecies in cells. With drugs targeting HBV polymerase (such as lamivudine, adefovir, and an acyclic nucleoside phosphonate), emergence of HBVresistant mutants develops during treatment [50]. An escape mutant was also selected for during shRNA treatment, discussed later [10].

\section{Successful RNAi Strategies against HBV}

Several RNAi effectors successfully downregulate HBV gene expression and replication in differing assay systems. A "very highly active" benchmark of $>95 \%$ reduction of extracellular viral particles from plasmid encoded HBV has been suggested for shRNAs warranting further development [7]. However, different experimental approaches and assays make quantitative comparison difficult. Assays for RNAi inhibition commonly used are (i) reporter gene assays, for example luciferase [14], (ii) reduction of viral RNAs from HBV derived from a plasmid in cultured cells [7], (iii) HBV-expressing transgenic mice or cells $[12,51]$, and (iv) hydrodynamically HBV-infected mice [9].

Analysis of characteristics of successful targets revealed different strategies of rational design for RNAi effectors, RNAi approaches, and mechanism of delivery. Nevertheless, these could be classified into 3 main groups: Group I: sequence conservation-based rational design-shRNA expression vectors (Pol II/III promoter) using a liposome delivery method; Group II: sequence conservation-based rational design-miRNA expression vectors (Pol II promoter) using a liposome delivery method; Group III: single siRNA programme prediction-shRNA expression vectors (Pol III promoter) using viral vector delivery methods (reviewed in $[8$, 9]). Successful target sequences and RNAi inhibitory effects of these 3 groups are indicated in Table 1. A summary of the effective target positions is shown in Figure 2.

\section{Variation in HBV Genotypes}

The $3.2 \mathrm{~kb}$ HBV genome is classified into eight main genotypes $(\mathrm{A}-\mathrm{H})$ with over $8 \%$ sequence diversity, with genotypes $\mathrm{A}-\mathrm{D}$ the most prevalent $[13,48,49]$. I is newly discovered but not ratified [54]. Therefore it is not surprising that there are few regions conserved across all genotypes $[7,13]$. These regions are often in sites of functional conservation in the RNAs or DNA, including the epsilon RNA and enhancer DNA elements (Figure 2). Some are in sites of overlapping genes, where the two open reading frames constrain sequence. Sun et al. [7] identified only one sequence of 17 bases conserved across representatives of all genotypes (1181-1897). They therefore used lesser stringency criteria of $\geq 98 \%$ or $\geq 95 \%$ identity for $\geq 15$ or more bases across genotypes $\mathrm{A}-\mathrm{D}$ as a practical limit to identify likely RNAi targets, this being about $\sim 300-500$ bases of the genome. They targeted 19 conserved sites in genotype D (ayw) with part of a panel of 21 shRNAs. Many of these were effective (Table 1), including some within the structured RNA epsilon element. The most effective target site in genotype D from that series (sh10) overlaps a conserved block but is not the most conserved target (1 variation in $A, B, E ; 2$ in $G, H)$. However, sh6, another very highly active shRNA, targets a block with no variation in genotypes $\mathrm{A}-\mathrm{H}$ (Table 1). In other reported studies that included conservation in design, some target better genotype A-C [14].

Zhang et al. identified 40 shRNA targets with conservation between genotypes A-I using an alignment of 327 representative sequences from Genbank as a guide [13]. They tested the shRNA against genotypes A-D, and I. The most effective four (B245, B376, B1581, and B1789) were able to reduce HBV production by up to $90 \%$ in both in vitro transfection and in vivo hydrodynamic model systems (Table 1).

As there was some dissimilarity in target design, there is not good concordance between the targets chosen in the studies of Zhang et al. [13] and Sun et al. [7]. However, some of the best sites had similar sites in the complementary study, but for these there was not good concordance in degree of inhibition. For example B245 (245-265), the best target from Zhang et al., is similar to sh4 (247-257) one of the weakest in Sun et al. Conversely the effective sh6 (416-434) is similar to B415 (415-435) but B415 had only a weak inhibitory effect. This may reflect subtle differences in targets, the vectors or 


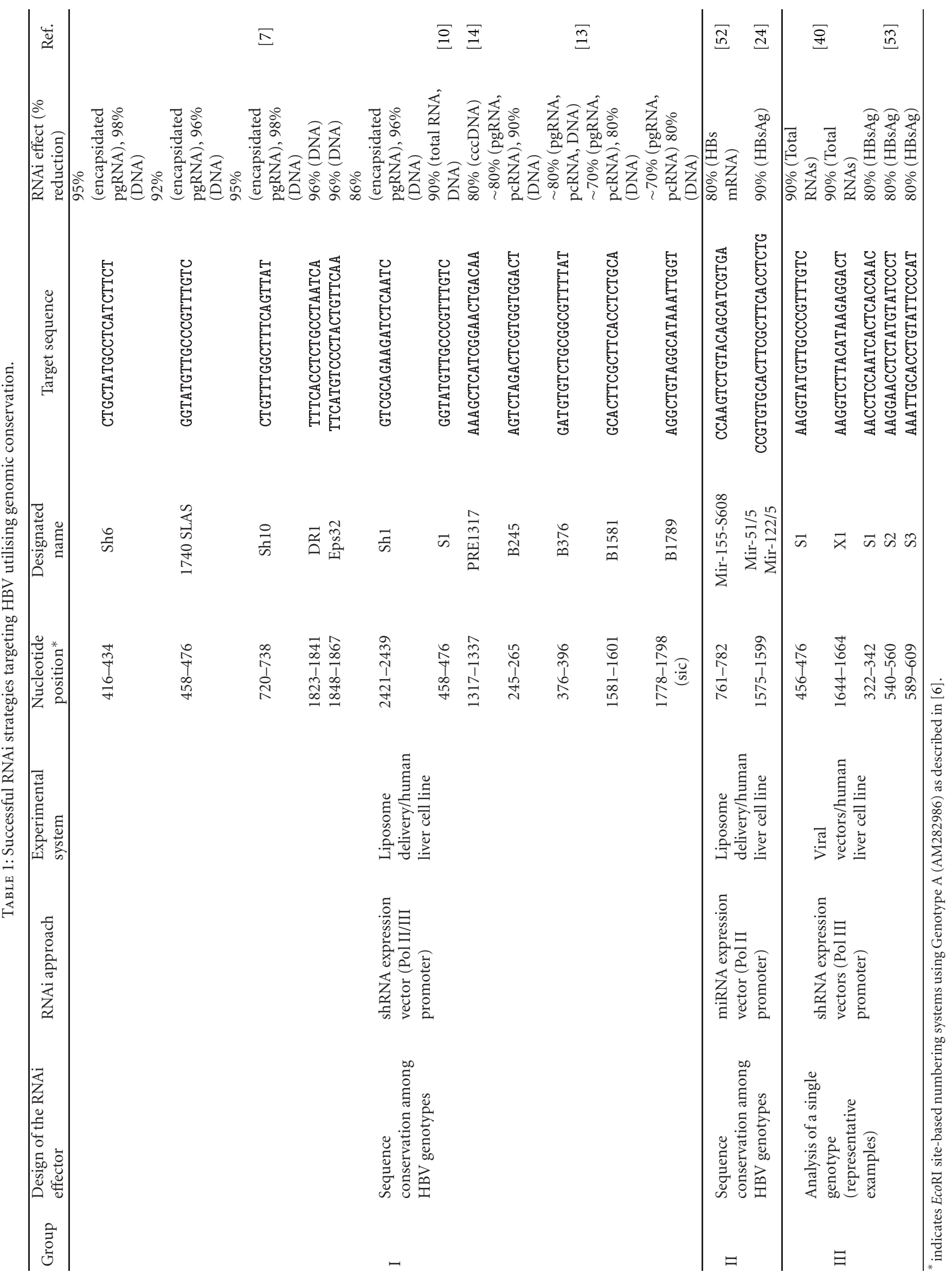


assays used, and supports a need for common standards within experiments as suggested by Sun et al.

\section{Rare Variants-Could These Be Selected for by RNAi?}

HBV polymerase has a high error rate producing many mutants-most of these with lower replication fitness. There are over 2,500 full-length HBV genomes in the Genbank database, and other databases contain rare sequence variations and mutations $[50,55,56]$. These may represent true replication competent variants, rare nonfunctional RNAs in the infected cells, or PCR or sequencing errors. As it is difficult to distinguish between these possibilities, redesigning an RNAi strategy to avoid them would be difficult.

In the recent study by Sun et al. they also tested the several shRNAs designed for HBV against Wooley monkey HBV (WMHBV). In WMHBV target sites differed in 14 positions. Some single variations retained partial activity (e.g., sh6 still inhibited to 14\%), but most abolished it, as did single mutations in the shRNA [7]. This is consistent with the idea that there must be an exact match between shRNA and target. However, it might be expected that variation in the $5^{\prime}$ end of the target, as was the case with sh6, would be more tolerated based on other RNAi studies, but this has not been systematically tested for HBV targets.

One study has found a resistant mutation that could be selected for following shRNA treatment in cell culture [10]. The shRNA used was designed to target a conserved site in all except genotype $\mathrm{H}(456-476)$ and was found to be effective in A-C. However a rare mutation in genotype C could be selected for by shRNA treatment in cultured cells. This mutation was silent with respect to both $\mathrm{S}$ and Pol overlapping protein coding and found in only one chronic carrier. Emergence of this type of shRNA-induced resistance has been seen for other viruses, notably $\operatorname{HIV}[57,58]$. In some cases like this a redundant pool of shRNAs containing a mix at a single position might be effective, for example, where a single position was changed, T472C or T472G in genotype $\mathrm{H}[10]$. To our knowledge this approach has not been used for HBV variants.

Deep sequencing using next (or new) generation (NGS) sequencing technologies allows the sequencing of many members of the HBV quasispecies infecting a single human. For HBV several studies have been done to investigate the emergence of mutations due to drugs targeting proteins [59-61]. Low prevalence drug resistance mutations could be detected by NGS with a greater sensitivity than PCR in both naïve and treated patients. These initial studies focused on mutations that change one of 288 RT amino acids. Many novel changes were detected-71 present in over $1 \%$ of the 2,800-18,000 sequences from each patient. Data from such deep sequencing studies would also be useful in design of RNAi against conserved sites, if possible rare tolerated mutations or polymorphisms should be avoided in target sites.

\section{Conclusion and Directions for Further Studies}

Most current assays for $\mathrm{HBV}$ replication more closely mimic acute HBV infection, with a single infecting genotype. In chronic infection the system where the virus is represented by a quasispecies in the infected individual selection due to the RNAi might be different [13].

It is possible that the rare genotypes, for example $\mathrm{H}$, would require a genotype-specific RNAi combination. Highly effective RNAi that does not target conserved blocks would still be useful if the genotype of the target is known.

Rationally designed RNAs, targeted in combinations [40, 62-64], delivered by "state-of-the-art" vectors could be an effective anti-HBV treatment $[4,5,8,9]$. Such design strategies would need to take into account conservation in the HBV genome. Several groups have identified effective target sites that are beginning to fulfill these criteria, and these will provide tools for further development.

\section{Acknowledgments}

N. Panjaworayan is funded by The Kasetsart University Research and Development Institute Grant (Vor Tor Dor 45.53) and a Research Grant for New Scholars (cofunded by TRF and CHE: MRG5380104). Part of this work was supported by a NZ Health Research Council Grant (05/195) to Warren Tate, Elizabeth Poole and CMB.

\section{References}

[1] N. S. Lee, T. Dohjima, G. Bauer et al., "Expression of small interfering RNAs targeted against HIV-1 rev transcripts in human cells," Nature Biotechnology, vol. 20, no. 5, pp. 500-505, 2002.

[2] J. M. Jacque, K. Triques, and M. Stevenson, "Modulation of HIV-1 replication by RNA interference," Nature, vol. 418, no. 6896, pp. 435-438, 2002.

[3] J. A. Wilson, S. Jayasena, A. Khvorova et al., "RNA interference blocks gene expression and RNA synthesis from hepatitis C replicons propagated in human liver cells," Proceedings of the National Academy of Sciences of the United States of America, vol. 100, no. 5, pp. 2783-2788, 2003.

[4] J. S. Pan, X. Z. Wang, and J. L. Ren, "Long-term RNA interference and its application to hepatitis B virus," Journal of Digestive Diseases, vol. 10, no. 3, pp. 165-171, 2009.

[5] P. Arbuthnot, V. Longshaw, T. Naidoo, and M. S. Weinberg, "Opportunities for treating chronic hepatitis B and C virus infection using RNA interference," Journal of Viral Hepatitis, vol. 14, no. 7, pp. 447-459, 2007.

[6] P. Arbuthnot, "Harnessing RNA interference for the treatment of viral infections," Drug News and Perspectives, vol. 23, no. 6, pp. 341-350, 2010.

[7] D. Sun, C. Rösler, K. Kidd-Ljunggren, and M. Nassal, "Quantitative assessment of the antiviral potencies of 21 shRNA vectors targeting conserved, including structured, hepatitis B virus sites," Journal of Hepatology, vol. 52, no. 6, pp. 817-826, 2010.

[8] A. P. McCaffrey, "RNA interference inhibitors of hepatitis B virus," Annals of the New York Academy of Sciences, vol. 1175, pp. 15-23, 2009. 
[9] D. Grimm and M. A. Kay, "Therapeutic short hairpin RNA expression in the liver: viral targets and vectors," Gene Therapy, vol. 13 , no. 6 , pp. 563-575, 2006.

[10] H. L. Wu, L. I. R. Huang, C. C. Huang et al., "RNA interference-mediated control of hepatitis B virus and emergence of resistant mutant," Gastroenterology, vol. 128, no. 3, pp. 708-716, 2005.

[11] D. Grimm, K. L. Streetz, C. L. Jopling et al., "Fatality in mice due to oversaturation of cellular microRNA/short hairpin RNA pathways," Nature, vol. 441, no. 7092, pp. 537-541, 2006.

[12] D. Grimm, L. Wang, J. S. Lee et al., "Argonaute proteins are key determinants of RNAi efficacy, toxicity, and persistence in the adult mouse liver," Journal of Clinical Investigation, vol. 120, no. 9, pp. 3106-3119, 2010.

[13] Y. L. Zhang, T. Cheng, Y. J. Cai et al., "RNA Interference inhibits hepatitis B virus of different genotypes in vitro and in vivo," BMC Microbiology, vol. 10, article 214, 2010.

[14] N. Panjaworayan, S. Payungporn, Y. Poovorawan, and C. M. Brown, "Identification of an effective siRNA target site and functional regulatory elements, within the hepatitis B virus posttranscriptional regulatory element," Virology Journal, vol. 7, atricle 216, 2010.

[15] J. M. Bosher and M. Labouesse, "RNA interference: genetic wand and genetic watchdog," Nature Cell Biology, vol. 2, no. 2, pp. E31-E36, 2000.

[16] N. Ramadan, I. Flockhart, M. Booker, N. Perrimon, and B. Mathey-Prevot, "Design and implementation of highthroughput RNAi screens in cultured Drosophila cells," Nature Protocols, vol. 2, no. 9, pp. 2245-2264, 2007.

[17] A. R. Naqvi, MD. N. Islam, N. R. Choudhury, and Q. M. R. Haq, "The fascinating world of RNA interference," International Journal of Biological Sciences, vol. 5, no. 2, pp. 97 $117,2009$.

[18] D. P. Bartel, "MicroRNAs: genomics, biogenesis, mechanism, and function," Cell, vol. 116, no. 2, pp. 281-297, 2004.

[19] A. Khvorova, A. Reynolds, and S. D. Jayasena, "Functional siRNAs and miRNAs exhibit strand bias," Cell, vol. 115, no. 2, pp. 209-216, 2003.

[20] D. S. Schwarz, G. Hutvágner, T. Du, Z. Xu, N. Aronin, and P. D. Zamore, "Asymmetry in the assembly of the RNAi enzyme complex," Cell, vol. 115, no. 2, pp. 199-208, 2003.

[21] S. Parrish, J. Fleenor, S. Xu, C. Mello, and A. Fire, "Functional anatomy of a dsRNA trigger: differential requirement for the two trigger strands in RNA interference," Molecular Cell, vol. 6, no. 5, pp. 1077-1087, 2000.

[22] S. M. Elbashir, J. Harborth, W. Lendeckel, A. Yalcin, K. Weber, and T. Tuschl, "Duplexes of 21-nucleotide RNAs mediate RNA interference in cultured mammalian cells," Nature, vol. 411, no. 6836, pp. 494-498, 2001.

[23] A. Fire, S. Xu, M. K. Montgomery, S. A. Kostas, S. E. Driver, and C. C. Mello, "Potent and specific genetic interference by double-stranded RNA in Caenorhabditis elegans," Nature, vol. 391, no. 6669, pp. 806-811, 1998.

[24] A. Ely, T. Naidoo, S. Mufamadi, C. Crowther, and P. Arbuthnot, "Expressed anti-HBV primary microRNA shuttles inhibit viral replication efficiently in vitro and in vivo," Molecular Therapy, vol. 16, no. 6, pp. 1105-1112, 2008.

[25] M. H. Han, S. Goud, L. Song, and N. Fedoroff, "The Arabidopsis double-stranded RNA-binding protein HYL1 plays a role in microRNA-mediated gene regulation," Proceedings of the National Academy of Sciences of the United States of America, vol. 101, no. 4, pp. 1093-1098, 2004.

[26] Y. Lee, C. Ahn, J. Han et al., "The nuclear RNase III Drosha initiates microRNA processing," Nature, vol. 425, no. 6956, pp. 415-419, 2003.
[27] K. M. Bollman, M. J. Aukerman, M. Y. Park, C. Hunter, T. Z. Berardini, and S. R. Poethig, "HASTY, the Arabidopsis ortholog of exportin 5/MSN5, regulates phase change and morphogenesis," Development, vol. 130, no. 8, pp. 1493-1504, 2003.

[28] S. M. Beverley, "Protozomics: trypanosomatid parasite genetics comes of age," Nature Reviews Genetics, vol. 4, no. 1, pp. $11-19,2003$

[29] V. Ambros, B. Bartel, D. P. Bartel et al., "A uniform system for microRNA annotation," RNA, vol. 9, no. 3, pp. 277-279, 2003.

[30] P. T. Nelson, A. G. Hatzigeorgiou, and Z. Mourelatos, "miRNP:mRNA association in polyribosomes in a human neuronal cell line," RNA, vol. 10, no. 3, pp. 387-394, 2004.

[31] D. G. Hendrickson, D. J. Hogan, H. L. McCullough et al., "Concordant regulation of translation and mRNA abundance for hundreds of targets of a human microRNA," PLoS Biology, vol. 7, no. 11, Article ID e1000238, 2009.

[32] H. Guo, N. T. Ingolia, J. S. Weissman, and D. P. Bartel, "Mammalian microRNAs predominantly act to decrease target mRNA levels," Nature, vol. 466, no. 7308, pp. 835-840, 2010.

[33] D. Jones, "Teaming up to tackle RNAi delivery challenge," Nature Reviews Drug Discovery, vol. 8, no. 7, pp. 525-526, 2009.

[34] M. Sioud and D. R. Sørensen, "Cationic liposome-mediated delivery of siRNAs in adult mice," Biochemical and Biophysical Research Communications, vol. 312, no. 4, pp. 1220-1225, 2003.

[35] L. Li and Y. U. Shen, "Overcoming obstacles to develop effective and safe siRNA therapeutics," Expert Opinion on Biological Therapy, vol. 9, no. 5, pp. 609-619, 2009.

[36] J. K. Watts, N. Choubdar, K. Sadalapure et al., " 2 'Fluoro- $4^{\prime}$-thioarabino-modified oligonucleotides: conformational switches linked to siRNA activity," Nucleic Acids Research, vol. 35, no. 5, pp. 1441-1451, 2007.

[37] T. Shiraishi, R. Hamzavi, and P. E. Nielsen, "Subnanomolar antisense activity of phosphonate-peptide nucleic acid (PNA) conjugates delivered by cationic lipids to HeLa cells," Nucleic Acids Research, vol. 36, no. 13, pp. 4424-4432, 2008.

[38] T. Hosono, H. Mizuguchi, K. Katayama et al., "Adenovirus vector-mediated doxycycline-inducible RNA interference," Human Gene Therapy, vol. 15, no. 8, pp. 813-819, 2004.

[39] L. Deng, G. Li, L. Xi et al., "Hepatitis B virus inhibition in mice by lentiviral vector mediated short hairpin RNA," $B M C$ Gastroenterology, vol. 9, article 73, p. 73, 2009.

[40] Y. Sun, Z. Li, L. Li, J. Li, X. Liu, and W. Li, "Effective inhibition of hepatitis B virus replication by small interfering RNAs expressed from human foamy virus vectors," International Journal of Molecular Medicine, vol. 19, no. 4, pp. 705-711, 2007.

[41] G. Doitsh and Y. Shaul, "A long HBV transcript encoding pX is inefficiently exported from the nucleus," Virology, vol. 309, no. 2, pp. 339-349, 2003.

[42] D. Siolas, C. Lerner, J. Burchard et al., "Synthetic shRNAs as potent RNAi triggers," Nature Biotechnology, vol. 23, no. 2, pp. 227-231, 2005.

[43] A. V. Vlassov, B. Korba, K. Farrar et al., "shRNAs targeting hepatitis C: effects of sequence and structural features, and comparison with siRNA," Oligonucleotides, vol. 17, no. 2, pp. 223-236, 2007.

[44] A. Reynolds, D. Leake, Q. Boese, S. Scaringe, W. S. Marshall, and A. Khvorova, "Rational siRNA design for RNA interference," Nature Biotechnology, vol. 22, no. 3, pp. 326-330, 2004.

[45] J. C. Giering, D. Grimm, T. A. Storm, and M. A. Kay, "Expression of shRNA from a tissue-specific pol II promoter 
is an effective and safe RNAi therapeutic," Molecular Therapy, vol. 16, no. 9, pp. 1630-1636, 2008.

[46] A. Chen, Y. F. Kao, and C. M. Brown, "Translation of the first upstream ORF in the hepatitis B virus pregenomic RNA modulates translation at the core and polymerase initiation codons," Nucleic Acids Research, vol. 33, no. 4, pp. 1169-1181, 2005.

[47] C. Seeger and W. S. Mason, "Hepatitis B virus biology," Microbiology and Molecular Biology Reviews, vol. 64, no. 1, pp. 51-68, 2000.

[48] H. Norder, A. M. Couroucé, P. Coursaget et al., "Genetic diversity of hepatitis B virus strains derived worldwide: genotypes, subgenotypes, and HBAg subtypes," Intervirology, vol. 47, no. 6, pp. 289-309, 2004.

[49] S. Schaefer, "Hepatitis B virus taxonomy and hepatitis B virus genotypes," World Journal of Gastroenterology, vol. 13, no. 1, pp. 14-21, 2007.

[50] S.-Y. Rhee, S. Margeridon-Thermet, M. H. Nguyen et al., "Hepatitis B virus reverse transcriptase sequence variant database for sequence analysis and mutation discovery," Antiviral Research, vol. 88, no. 3, pp. 269-275, 2010.

[51] C. C. Chen, C. P. Sun, H. I. Ma et al., "Comparative study of anti-hepatitis B virus RNA interference by double-stranded adeno-associated virus serotypes 7, 8, and 9," Molecular Therapy, vol. 17, no. 2, pp. 352-359, 2009.

[52] Y. U. F. Gao, L. I. Yu, W. Wei, J. B. Li, Q. L. Luo, and J. I. L. Shen, "Inhibition of hepatitis B virus gene expression and replication by artificial microRNA," World Journal of Gastroenterology, vol. 14, no. 29, pp. 4684-4689, 2008.

[53] J. Peng, Y. Zhao, J. Mai et al., "Inhibition of hepatitis B virus replication by various RNAi constructs and their pharmacodynamic properties," Journal of General Virology, vol. 86, no. 12, pp. 3227-3234, 2005.

[54] F. Kurbanov, Y. Tanaka, A. Kramvis, P. Simmonds, and M. Mizokami, "When should "I" consider a new hepatitis B virus genotype?" Journal of Virology, vol. 82, no. 16, pp. 8241-8242, 2008.

[55] M. Buti, F. Rodriguez-Frias, R. Jardi, and R. Esteban, "Hepatitis $\mathrm{B}$ virus genome variability and disease progression: the impact of pre-core mutants and HBV genotypes," Journal of Clinical Virology, vol. 34, supplement 1, pp. S79-S82, 2005.

[56] S. M. Jazayeri, S. M. Alavian, and W. F. Carman, "Hepatitis B virus: origin and evolution," Journal of Viral Hepatitis, vol. 17, no. 4, pp. 229-235, 2010.

[57] E. M. Westerhout, M. Ooms, M. Vink, A. T. Das, and B. Berkhout, "HIV-1 can escape from RNA interference by evolving an alternative structure in its RNA genome," Nucleic Acids Research, vol. 33, no. 2, pp. 796-804, 2005.

[58] D. Boden, O. Pusch, and B. Ramratnam, "Overcoming HIV-1 resistance to RNA interference," Frontiers in Bioscience, vol. 12, pp. 3104-3116, 2007.

[59] M. Solmone, D. Vincenti, M. C. F. Prosperi, A. Bruselles, G. Ippolito, and M. R. Capobianchi, "Use of massively parallel ultradeep pyrosequencing to characterize the genetic diversity of hepatitis B virus in drug-resistant and drug-naive patients and to detect minor variants in reverse transcriptase and hepatitis B S antigen," Journal of Virology, vol. 83, no. 4, pp. 1718-1726, 2009.

[60] S. Margeridon-Thermet, N. S. Shulman, A. Ahmed et al., "Ultra-deep pyrosequencing of hepatitis b virus quasispecies from nucleoside and nucleotide reverse-transcriptase inhibitor (NRTI)-treated patients and NRTI-naive patients," Journal of Infectious Diseases, vol. 199, no. 9, pp. 1275-1285, 2009.
[61] T. Pollicino, G. Isgrò, R. Di Stefano et al., "Variability of reverse transcriptase and overlapping $S$ gene in hepatitis $B$ virus isolates from untreated and lamivudine-resistant chronic hepatitis B patients," Antiviral Therapy, vol. 14, no. 5, pp. 649$654,2009$.

[62] K. L. Wu, X. Zhang, J. Zhang et al., "Inhibition of Hepatitis $\mathrm{B}$ virus gene expression by single and dual small interfering RNA treatment," Virus Research, vol. 112, no. 1-2, pp. 100$107,2005$.

[63] Z. Li, M. L. He, H. Yao et al., "Inhibition of HBV replication and gene expression in vitro and in vivo with a single AAV vector delivering two shRNA molecules," BMB Reports, vol. 42, no. 1, pp. 59-64, 2009.

[64] X. M. Xin, G. Q. Li, Y. Y. Jin, M. Zhuang, and D. I. Li, "Combination of small interfering RNAs mediates greater suppression on hepatitis B virus cccDNA in HepG2.2.15 cells," World Journal of Gastroenterology, vol. 14, no. 24, pp. 38493854, 2008. 


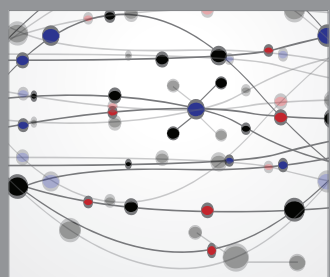

The Scientific World Journal
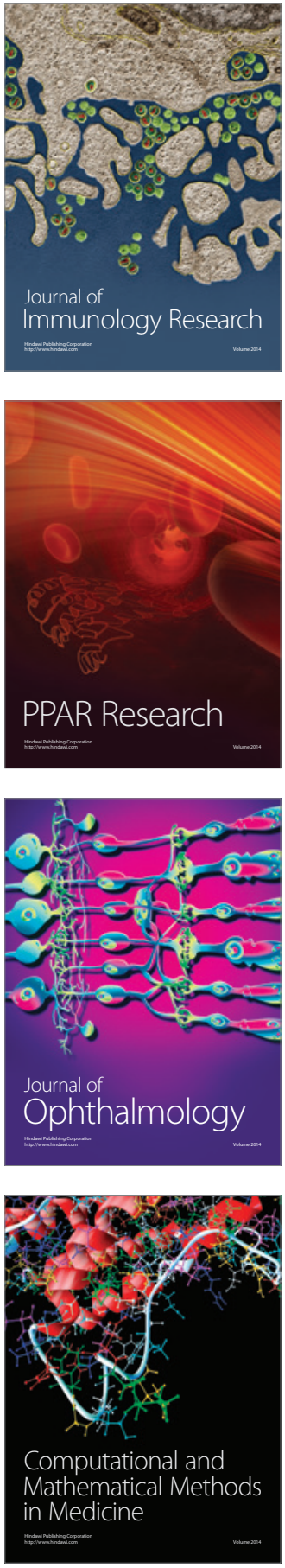

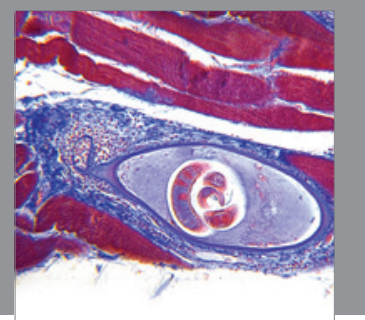

Gastroenterology

Research and Practice
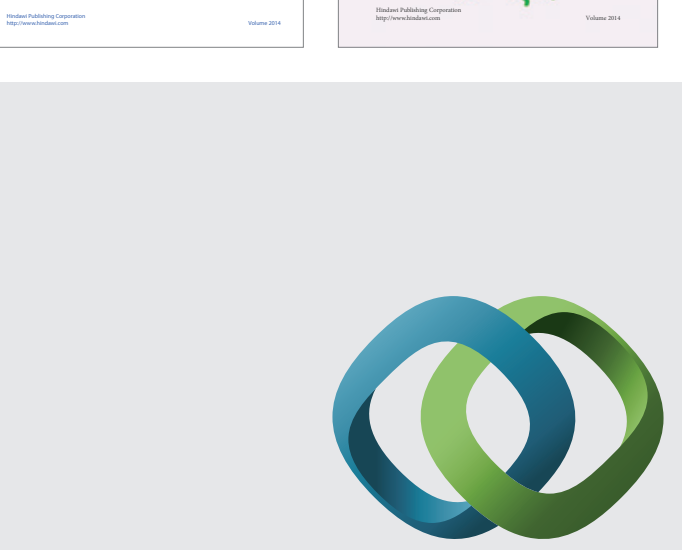

\section{Hindawi}

Submit your manuscripts at

http://www.hindawi.com
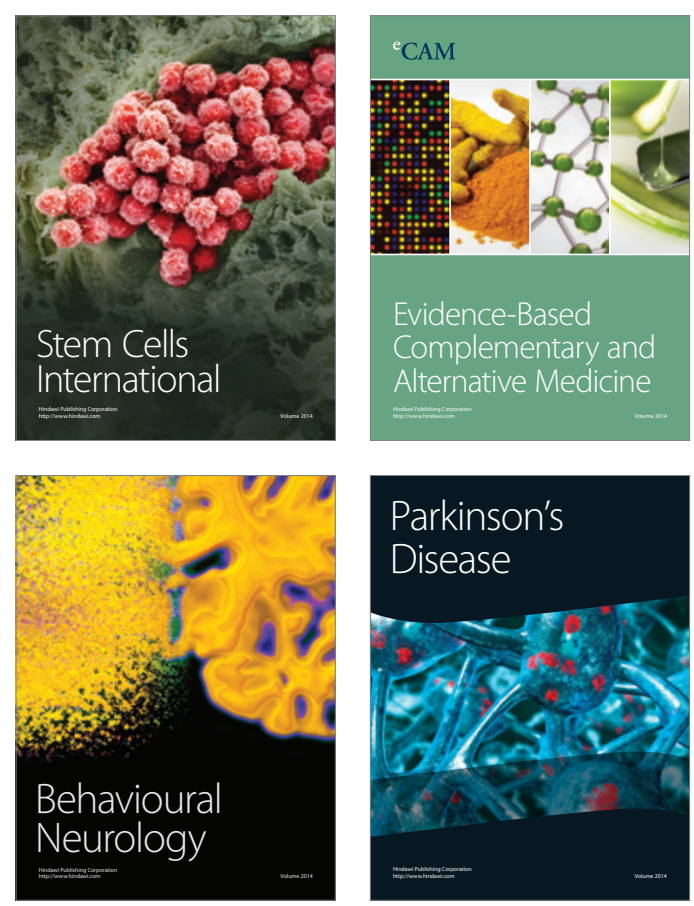

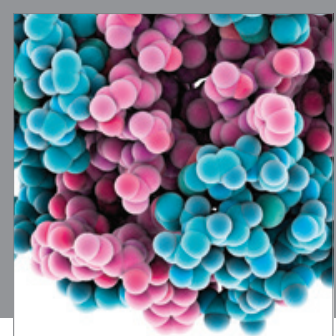

Journal of
Diabetes Research

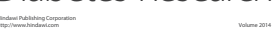

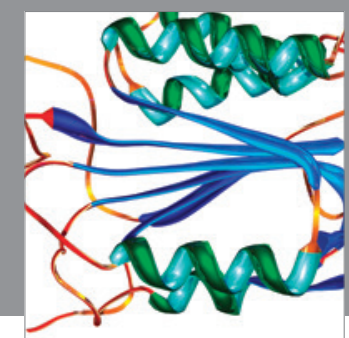

Disease Markers
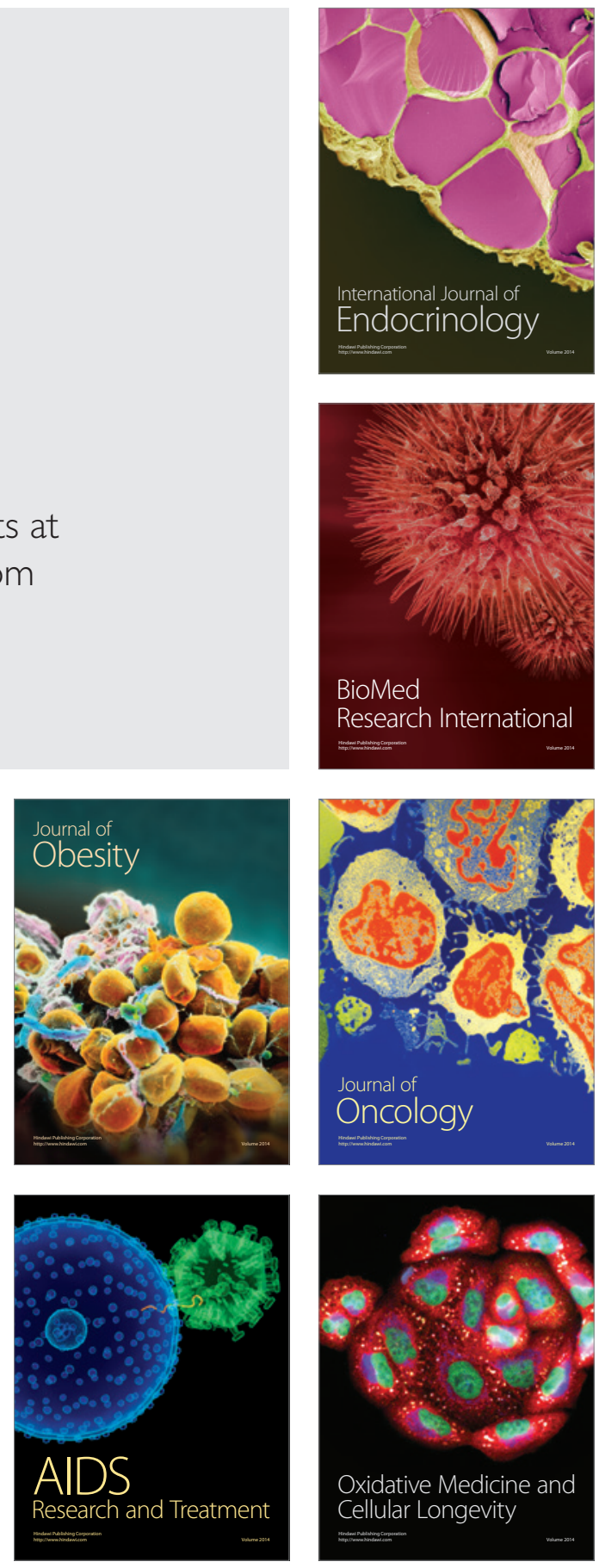